\title{
Periprosthetic Fractures after Total Knee Arthroplasty: the Influence of Pre-Operative Mechanical Factors versus Intraoperative Factors
}

\author{
Zainul-Abidin S, FRCS (Orth), Lim BTJ, FRCS (Orth), Bin-Abd-Razak HR, FRCS (Orth), Gatot C, MBBS, \\ Allen JC*, PhD, Koh JSB, FRCS, Howe TS, FRCS \\ Department of Orthopaedic Surgery, Singapore General Hospital, Singapore \\ *Centre for Quantitative Medicine, DukeNUS Medical School, Singapore
}

\author{
(c) (1) \\ This is an open-access article distributed under the terms of the Creative Commons Attribution License, which permits unrestricted use, \\ distribution, and reproduction in any medium, provided the original work is properly cited \\ Date of submission: 9th April 2019 \\ Date of acceptance: 20th June 2019
}

\begin{abstract}
Introduction: Periprosthetic fractures are a devastating complication following total knee arthroplasty. Little is known about the effect of mechanical factors on the incidence of periprosthetic fractures. The aim of this study was to examine the correlation between pre-operative mechanical factors, like side of surgery, coronal alignment and pre-operative range of motion and intra-operative factors, and the incidence of a periprosthetic fracture, following primary total knee arthroplasty (TKA).

Materials and Methods: Forty-two patients with periprosthetic fractures (PPF) after primary TKA were identified from our hospital arthroplasty registry. These patients were matched two-to-one for gender and age at primary knee arthroplasty to 84 patients without PPF. The incidence of periprosthetic fracture with regards to laterality, coronal alignment and pre-operative range of motion was analysed. Intra-operative factors like implant type, patellar resurfacing and notching were also analysed using logistic regression.

Results: Coronal alignment, pre-operative range of motion and patella resurfacing were not significant predictors of periprosthetic fractures. Anterior femoral notching was found to be significantly higher in the fracture group with an odds ratio of 17. Left sided surgery was also significantly higher in the periprosthetic fracture group.

Conclusion: Periprosthetic fractures are 17 times more likely to occur in a knee with anterior femoral notching. Preoperative factors like coronal alignment and poor preoperative range of motion do not seem to increase the risk of periprosthetic fractures after TKA.
\end{abstract}

Key Words: periprosthetic fracture, total knee arthroplasty, notching, valgus knee, range of motion

\section{INTRODUCTION}

Total Knee Arthroplasty (TKA) is widely regarded as a safe, effective and rewarding procedure, with patients consistently reporting excellent satisfaction rates ranging from $78 \%$ to $94 \%{ }^{1}$. Periprosthetic fractures (PPF) however, are a devastating complication for both the patients and surgeons, potentially rendering the patient immobile, drastically reducing their function and also frequently resulting in multiple surgical procedures, increased duration of hospital stay as well as additional hospital expense. The minority of TKA patients who suffer from periprosthetic fractures unfortunately eventually end up having poorer functional outcome scores and satisfaction rates ${ }^{2}$.

Preoperative risk factors that have been reported in the literature thus far include, previous revision TKA, prolonged steroid use, rheumatoid arthritis, advanced age, female gender, neurologic disorders and, controversially, the presence of anterior femoral notching ${ }^{3-5}$. However, the effect of pre-operative factors on periprosthetic fractures is less well documented. Limited analysis of the outcomes of patients with poor pre-operative range of motion (ROM) undergoing knee arthroplasty found that there was a much higher complication rate of $41 \%$ and a high revision rate of $18.5 \%$, including revisions secondary to $\mathrm{PPF}^{6}$. Other potential mechanical factors such as coronal leg alignment (pre-operative varus or valgus deformity) and leg side dominance on the incidence of periprosthetic fractures have not been studied in depth though some studies suggested that varus malalignment was associated with periprosthetic fractures?.

Our null hypothesis is that mechanical factors such as coronal alignment, leg dominance and pre-operative range of motion are similar between patients with and without

Corresponding Author: Suraya Zainul-Abidin, Department of Orthopaedic Surgery, Singapore General Hospital, Outram Road, Singapore 169608

Email: Suraya@doctors.org.uk 
periprosthetic fractures. Our study aimed to compare preoperative mechanical factors (including coronal leg alignment and pre-operative range of motion) between patients sustaining periprosthetic fractures after TKR against a gender and age-matched control group with no periprosthetic fracture. These factors were also analysed against the potential effects of intra-operative technical confounders such as anterior femoral notching and implant constraint.

\section{MATERIALS AND METHODS}

The 'Case' group (or 'fracture' group) of 42 patients with periprosthetic fracture following primary TKA was identified from prospectively collected data in the hospital arthroplasty registry between 2000 and 2015. All patients underwent primary total knee arthroplasty by a fellowshiptrained orthopaedic specialist surgeon and were placed on a standardised postoperative arthroplasty clinical pathway and physiotherapy regime in our institution. All patients with a lower limb deformity not related to the primary surgery and/or neurological deficit impairing the function of the operated limb were excluded from the study.

These 42 patients were matched 2:1 for gender and age at primary knee arthroplasty to 84 patients without periprosthetic fracture after TKA ('Control' or 'non fracture' group). Pre-operative patient demographics (age, gender, $\mathrm{BMI}$, pre-operative range of motion, coronal alignment (varus or valgus deformity) and radiographs were analysed for all patients.

To determine coronal leg alignment and range of motion, all patients were assessed pre-operatively by a specially trained group of physiotherapists. Knee alignment was measured using photogrammetry: in this technique, bony processes were identified by manual palpation. The anterior superior iliac spine (ASIS), centre of patella and talar head were identified in accordance with the recommendations of Gross et $a l^{8}$. Spherical markers were attached at these anatomical points. The patients were then photographed. Frontal views were analysed by our AutoCAD 2000 software to measure the angles between the thigh and lower leg segments. In the frontal views, varus / valgus angles of the knee were drawn using two straight lines, one joining the ASIS to the centre of the patella, and the other joining the centre of the patella to the talar head ${ }^{9}$. Range of motion of the knee was measured using a goniometer, and measuring the angles at the knee during maximal flexion and extension.

All patients were planned for a primary total knee replacement via a parapatellar approach using either cruciate-retaining or posterior-stabilized implants, with cementing of the prostheses in all patients. Not all patellae were resurfaced and this was determined by surgeon's preference as well as the size of the patella intraoperatively. All patients had mechanical and/or chemo - prophylaxis to prevent venous thromboembolism post-operatively.

Postoperatively, patients were assessed by a physiotherapist daily. All patients were allowed to ambulate with a walking aid by the second postoperative day and were discharged home once they were able to actively flex the knee to 90 degrees, to perform unassisted straight-leg raising, to ambulate independently and to climb stairs (if they were required to do so at their homes). Patients who required additional rehabilitation were discharged to a community rehabilitation hospital. All patients underwent outpatient physiotherapy until their progress was deemed satisfactory for discharge.

Patients with periprosthetic fractures were contacted by telephone retrospectively to enquire on their leg dominance ("Which leg do you use first to climb up stairs?"). Leg dominance data was available for 33 out of the 42 patients. With regards to anterior femoral notching, radiographs of all the patients were reviewed by three of the authors (SZA, LBTJ and CG). The presence of anterior femoral notching in post-operative radiographs was ascertained and graded according to the Tayside Classification: Grade I (violation of the outer table of the anterior femoral cortex), Grade II (violation of the outer and inner table to the centre of the femoral cortex), Grade III (violation of up to $25 \%$ of the medullary canal) or Grade IV (violation of up to $50 \%$ of the medullary canal $)^{10}$.

Statistical analysis was carried out using paired t-test for continuous variables between the study and control group. The incidence of periprosthetic fractures with regard to fixed flexion deformity (FFD), flexion limitation, varus malalignment or valgus malalignment was analysed using a chi-squared test. Implant type, the presence of femoral notching, patellar resurfacing and proportion of RA versus OA were also analysed using the Chi-squared test.

Variables of patient demographics, ROM, side of surgery and coronal alignment were entered into a univariate logistic regression model. Variables with $\mathrm{p}$-value $<0.2$ were then entered into a multivariate logistic regression model in a stepwise manner. A variable was considered to be a significant predictor if its odds ratio was significant at $p<$ 0.05 after multiple regression analysis. All statistical analyses were performed using SPSS version 21 (SPSS, Inc., Chicago, Illinois) in consultation with a biostatistician (AJC).

Power analysis was performed to ensure that the study sample size was adequate. A post hoc power analysis using 126 patients as the sample size (total number of patients in the study), and an alpha value of 0.05 showed a power of $100 \%$. This study was designed as a retrospective casecontrol study. This study was exempted from IRB ethical approval as no patients were directly involved in the study and all data was anonymised. 


\section{RESULTS}

There were 42 patients with periprosthetic fractures following primary TKA (37 female and five male). These patients were matched 2:1 for age at TKA and gender to 84 patients (74 female and 10 male). Patient demographics are shown in Table I.

There was no difference in mean age at primary TKA for both groups $(68.9 \pm 8.2$ years $)$. BMI for both groups of patients was also not significantly different $(28.4 \pm 4.8$ for PPF group vs $27.5 \pm 3.9$ for control group, $\mathrm{p}=0.425$ ). Of the 42 PPFs, 40 were femoral fractures (supracondylar region of distal femur) whilst two were tibial fractures. Mean time period from primary TKA to occurrence of periprosthetic fracture was $22.8 \pm 30.5$ months, with 29 early ( less than two years after surgery) and 12 late fractures (more than two years after surgery).

Low velocity injury was the cause for all the peri-prosthetic fractures: 21 patients had fallen on slippery floor, 12 patients had tripped on uneven grounds/stairs and nine patients had twisted their knee while getting up from a lying/sitting position. Four patients also had a history of cerebrovascular accident, which could have predisposed to the falls.

The mean age of the 42 patients at the time of fracture was 71 years. In the periprosthetic fracture group, 39 patients were diagnosed with osteoarthritis, two with rheumatoid arthritis and one with avascular necrosis. In the non-fracture group, 83 patients had osteoarthritis whilst only one had rheumatoid arthritis. There was no statistically significant difference between the proportion of osteoarthritis and rheumatoid arthritis patients in both groups (Chi-square statistic $1.60, \mathrm{p}$-value $=0.206)$. From radiological assessments, there were 10 patients in the PPF group who had anterior femur notching after their primary TKA. Based on Tayside's classification, there were four patients with grade I notching, three patients with grade II notching and three patients with Grade III notching. Only three of the ten patients had fracture through their notching (two patients had Grade I notching and one had Grade III notching), which was not significant $(\mathrm{p}=0.073)$. Only three patients in the nonfracture group were found to have anterior femoral notching. Univariate logistic regression showed this to be significantly different between the fracture and non-fracture group $(\mathrm{p}=0.0019$, odds ratio 10.66). On multiple logistic regression, notching remained highly significant at $\mathrm{p}=0.0008$, with an odds ratio of 17.43 .

For the fracture group, the types of implants used were: 27 cruciate- retaining, 14 posterior-stabilised and one constrained knee (due to femoral notching). For the nonfracture group, there were 25 cruciate retaining implants, 57 posterior-stabilised implants and two constrained knee implants used (also due to femoral notching). There were significantly more posterior-stabilised implants in the nonfracture group (Chi-square statistic 14.01, $\mathrm{p}=0.000182$ ).
Of the 42 knees in the fracture group, 17 had the patella resurfaced whilst 25 of the patellae were not. Of the 84 patellae in the non-fracture group, 29 were resurfaced whilst 55 of the patella were not resurfaced. This was not found to be significantly different between the two groups (Chisquare statistic $0.3675, \mathrm{p}=0.544$ ).

Patients in the Peri-Prosthetic Fracture (PPF) group were found to have a mean fixed flexion deformity (FFD) of 9.3。 compared to $8.1^{\circ}$ in the control group. Univariate analysis revealed that pre-operative FFD was not significantly different between the PPF and control group, with p-value = 0.240. The mean pre-operative flexion in patients with PPF was $112.4 \circ$ compared to $116.7 \circ$ for the control group. This was also found to be non-significant on univariate analysis, with p-value of 0.503 .

The side of surgery was found to be a significant factor in predicting periprosthetic fractures, with a $\mathrm{p}$-value of 0.085 on univariate logistic regression. When entered into a multivariate logistic regression, side of surgery remained a significant factor predicting PPF ( $\mathrm{p}=0.0169$, odds ratio 2.88).

Twenty-six of $42(61.9 \%)$ patients in the PPF group had surgery on the left knee, whilst only 36 of 84 (42.9\%) nonfracture patients had their TKA done on the left knee. We attempted to obtain data for leg dominance, via our retrospective data collection (phone interviews), and periprosthetic fractures were found to have occurred in the non-dominant leg in 17 of 33 contactable patients (52\%). Five patients were deceased and four patients were uncontactable.

In terms of coronal alignment, 16 of $42(38 \%)$ patients in the PPF group had a valgus alignment pre-operatively, 25 (59.5\%) had varus alignment and 1 (2.3\%) patient was neutral. For the control group, 62 out of 82 patients $(73.8 \%)$ had varus alignment and $22(26.9 \%)$ had valgus alignment. Pre-operative coronal alignment (varus, valgus or neutral) trended towards significance with univariate analysis of coronal alignment revealing a p-value of 0.189 for valgus knees, however it was found to be non-significant when entered into a multivariate logistic regression analysis.

A subgroup analysis of early and late periprosthetic fractures was also done: Pre-op BMI, diagnosis of OA or RA, pre-op alignment and pre-op range of motion were not found to be significant predictors of late or early periprosthetic fractures. However, the side of surgery was more significant in the early PPF group ( $p=0.0407$ for the left side). Results of the statistical analysis and subgroup analyses are presented in Tables II and III.

\section{DISCUSSION}

The rates of TKA are increasing worldwide and correspondingly, rates of associated complications like 
Table I: Patient demographics and clinical details

\begin{tabular}{|lcc|}
\hline Patient Demographics and Clinical Details & Cases (PPF) & Controls (TKA with no PPF) \\
\hline Number of patients & 42 & 84 \\
Male & 5 & 10 \\
Female & 37 & 74 \\
Age at surgery & $68.9 \pm 8.2$ & $68.9 \pm 8.2$ \\
Body Mass Index & $28.4 \pm 4.8$ & $27.5 \pm 3.9$ \\
Side of surgery : Left & 26 & 36 \\
Side of Surgery : Right & 16 & 48 \\
Alignment : Varus & 25 & 62 \\
Alignment : Valgus & 16 & 22 \\
Alignment : Neutral & 1 & 0 \\
Pre-operative extension (mean) & $9.3 \pm 13.4$ & $8.1 \pm 8.7$ \\
Pre-operative flexion (mean) & $112.4 \pm 24.2$ & $116.7 \pm 17.6$ \\
Notching & 10 & 3 \\
CR implants & 27 & 57 \\
PS implants & 14 & 25 \\
Rheumatoid Arthritis & 2 & 1 \\
Osteoarthritis & 39 & 83 \\
\hline
\end{tabular}

Table II: Summary of statistical analysis

\begin{tabular}{|lcc|}
\hline & Cases & Controls \\
\hline FFD $<10$ & 25 & 48 \\
FFD $>10$ & 17 & 34 \\
Chi-Square statistic: $0.0112, \mathrm{p}=0.916$ & & 7 \\
Flexion $<90$ & 36 & 77 \\
Flexion $>90$ & & 57 \\
Chi-Square statistic: $0.1772, \mathrm{p}=0.3004$ & 14 & 25 \\
Posterior-Stabilised Implants & 27 & 83 \\
Cruciate-Retaining Implants & & 1 \\
Chi-Square Statistic: $14.01, \mathrm{P}=0.000182$ & 39 & 25 \\
Osteoarthritis & 2 & 59 \\
Rheumatoid Arthritis & & \\
Chi-Square statistic: $1.60, \mathrm{p}=0.206$ & 17 & P VALUE \\
Patella resurfaced & 24 & 0.186 \\
Patella not resurfaced & & 0.085 \\
Chi-Square statistic: $0.3675, \mathrm{p}=0.544$ & & 0.503 \\
& ODDS RATIO & 0.240 \\
UNIVARIATE LOGISTIC REGRESSION & 3.35 & 0.0019 \\
Valgus Alignment & 2.98 & \\
Left sided TKA & 0.45 & P VALUE \\
Pre-operative Extension & 1.38 & 0.0169 \\
Pre-operative flexion & 10.66 & 0.0008 \\
Notching & & \\
MULTIVARIATE LOGISTIC REGRESSION & ODDS RATIO & \\
Left Sided TKA & 2.88 & \\
Notching & 17.43 & \\
\hline
\end{tabular}

Table III: Subgroup analysis between early and late PPF

\begin{tabular}{|lc|}
\hline Univariate Logistic Regression & P Value \\
\hline Pre-op Alignment (Valgus vs Varus) & 0.305 \\
Pre-op BMI & 0.622 \\
Notching & 0.433 \\
Poor pre-op flexion & 0.310 \\
Poor Pre-op Extension (FFD) & 0.874 \\
Side & 0.0407 \\
\hline
\end{tabular}


periprosthetic fractures as well ${ }^{11}$. Studies have reported the incidence of periprosthetic fractures to range between 0.3 and $5.5 \%$ after primary TKA and even higher after revision TKA $^{12-13}$.

With the added morbidity, mortality and expense that are incurred associated with periprosthetic fractures, numerous studies have been done to delineate the risk factors that predispose to this dreaded complication.

Our study found that periprosthetic fractures after TKA tended to occur in the supracondylar region of the femur and were mainly early complications occurring within two years of index surgery. Previous studies have shown that many knee replacements fail early: $35.3 \%$ of revisions occur at less than two years, and up to $60 \%$ in the first five years, with the leading cause of failure being attributed to aseptic loosening, and also citing periprosthetic fractures as a major cause of revisions ${ }^{14}$.

Our results showed that pre-operative mechanical factors such as coronal alignment and pre-operative range of motion were not found to significantly influence periprosthetic fractures. Valgus knees have been reported to have a high rate of patella stress fracture and osteonecrosis as compared to non-valgus TKA (1-12\%) but our statistical analysis revealed that pre-operative valgus malalignment was not a significant risk factor for periprosthetic fracture. This finding is reassuring for arthroplasty surgeons who may be concerned about proceeding with TKA in a patient with valgus deformity ${ }^{15}$.

Our study also found that poor preoperative range of motion was not a significant predictor for periprosthetic fractures following primary TKA. It has been described in previous studies that poor pre-operative range of motion correlated with poor post-operative ROM and poorer functional outcomes. However, these poorer outcomes appeared not be the caused by PPF, but probably from poorer mobility and chronic pain from stiffness ${ }^{16}$.

Surgery done on the left knee was found to be significant at $\mathrm{p}=0.085$ on univariate logistic regression and still significant on a multivariate logistic regression $(\mathrm{p}=0.0169$, odds ratio 2.88). The relevance of side of surgery is currently unknown and more work is needed to determine if this correlates with the side of leg and what the implications are for performing surgery on the non-dominant leg.

A previous biomechanical study looking at muscle strength in dominant and non-dominant legs of females found that there is significant asymmetry in leg muscle strength, with stronger flexion in the non-dominant leg but stronger extension in the dominant $\operatorname{leg}^{17}$. There is little in the way of published research that investigates the significance of the side of surgery and subsequent rates of fractures or complications.
Young et al in $2013^{18}$ who investigated the impact of leg dominance on balance recovery, found that when subjected to a force which caused them to fall or become off balance, patients did not seem to fall more frequently if pulled towards their non-preferred side. However, this hypothesis was tested on normal patients and not those who had undergone surgery. Our study showed an association with the side of surgery (left side) and periprosthetic fractures; however, we were unable to ascertain whether this correlated with leg dominance.

Ten $(23.8 \%)$ of our patients with periprosthetic fractures had anterior femoral notching after primary TKA. This was found to be significantly higher than our control group $(p=0.0019$, odds ratio 10.66). Femoral notching has been found to significantly decrease distal femoral torsional load to failure in biomechanical studies ${ }^{19-20}$. However, some clinical studies looking at the effect of femoral notching have reported that there is no increase in the rate of occurrence of supracondylar fractures in the presence of notching ${ }^{10}$. Our study has shown anterior femoral notching to be a significant risk factor in periprosthetic fractures, in contradiction to the findings of Ritter et al in $2005^{21}$.

A major difference between the previous studies and our study is that previous studies identified a cohort of patients who underwent total knee replacement and subsequently followed them up to look for periprosthetic fractures, whereas our study initially identified a cohort of patients with periprosthetic fractures. Unfortunately, this methodology resulted in a very small number of periprosthetic fractures in existing studies (two fractures out of 1089 patients for Ritter et al, and three fractures out of 200 patients for Gujarati et $a l)^{21,10}$ - which may make the applicability of their findings limited.

In contrast, our study looked at a much larger cohort of 42 patients with periprosthetic fractures. When compared to the non-fracture group, it is clear that anterior femoral notching was higher in the fracture group, with a periprosthetic fracture being 17 times more likely to occur in a patient with anterior femoral notching.

A subgroup analysis for all these pre-operative mechanical factors was also done - pre-op BMI, diagnosis of OA or RA, pre-op alignment and pre-op range of motion were not found to be significant predictors of late or early periprosthetic fractures. However, the side of surgery was significantly higher in the early PPF group $(p=0.0407)$. This further confirmed that pre-operative factors like alignment, range of motion and BMI were not likely to have significant effects on periprosthetic fractures, but again highlighted this interesting finding of left sided TKAs being more likely to sustain fracture. 
Our study has several strengths: our matched patient groups (controlled for age, gender and BMI) allowed us to minimize confounding factors. In addition, study data was prospectively collected via our hospital registry and this allowed minimisation of recall bias. To our knowledge, there are few studies in current literature which analyse the association between side of surgery, coronal alignment and the incidence of PPF.

The limitations of our study is that of a relatively small sample size (though power analysis revealed our study to be adequately powered). In addition, not all patients had their bone mineral density measured and a large majority of our patients were female; and therefore likely to also have underlying osteoporosis. This bias was minimised by matching the control group for age and gender.

Additionally, both groups of patients were not heterogeneous in terms of implant type (CR or PS implant), resurfacing of patella or surgical approach, which could represent possible confounders. Although the numbers of CR and PS implants were significantly different between the fracture and nonfracture groups, previous studies have shown no difference in rate of periprosthetic fractures for CR or PS implants ${ }^{22}$.

\section{CONCLUSION}

Periprosthetic fractures are largely not influenced by preoperative mechanical factors like coronal alignment and range of motion, but rather by intra-operative technical events, specifically anterior femoral notching. Hence, careful emphasis on operative techniques could mitigate this devastating complication regardless of the pre-surgical mechanical alignment or range of motion.

\section{CONFLICT OF INTEREST}

The authors declare no potential conflict of interest.

\section{REFERENCES}

1. Bourne R, Chesworth B, Davies A, Mahomed N, Charron K. Patient satisfaction after total knee arthroplasty: Who is satisfied and who is not? Clin Orthop Relat Res. 2010; 468(1): 57-63.

2. Lizaur-Utrilla A, Miralles-Munoz F, Sanz-Reig J. Functional Outcome of total knee arthroplasty after periprosthetic distal femoral fracture. J Arthroplasty. 2013; 28(9): 1585-8.

3. Merkel K, Johnson E. Supracondylar fracture of the femur after total knee arthroplasty. J Bone Joint Surg Am. 1986; 68(1): 2943.

4. Meek R, Norwood T, Smith R, Brenkel I, Howie C. The risk of peri-prosthetic fracture after primary and revision total hip and knee replacement. J Bone Joint Surg Br. 2011; 93(1): 96-101.

5. Rorabeck C, Taylor J. Periprosthetic fractures of the femur complicating total knee arthroplasty. Orthop Clin North Am. 1999; 30(2): 265-77.

6. McAuley J, Harrer M, Ammeen D, Engh G. Outcome of knee arthroplasty in patients with poor preoperative range of motion. Clin Orthop Relat Res. 2002; (404): 203-7.

7. Parvizi J, Jain N, Schmidt A. Periprosthetic knee fractures. J Orthop Trauma. 2008; 22(9): 663-71.

8. Gross J, Fetto J, Rosen E. Exame musculoesqueletico. Porto Alegre: Artmed; 2000

9. Poletto P, Sato T, Carnaz L, Lobo Da Costa P, Gil Coury H. Do Individuals who present a static difference between the knees also present a difference during gait? Revista Brasilia de Fisioterapia. 2007; 11(1): 43-8.

10. Gujarati N, Putti A, Abboud R, Maclean J, Espley A, Kellett C. Risk of periprosthetic fracture after anterior notching. Acta Orthop. 2009; 5(80): 553-6.

11. Kim K, Egol K, Hozack W, Parvizi J. Periprosthetic fractures after total knee arthroplasties. Clin Ortho Relat Res. 2006; 446:16775.

12. Figgie M, Goldberg V, Figgie H, Sobel M. The results of treatment of supracondylar fracture above total knee arthroplasty. $J$ Arthroplasty. 1990; 5(3): 267-76.

13. Inglis A, Walker P. Revision of failed knee replacements using fixed-axis hinges. J Bone Joint Surg Br. 1991; 73(5): 57-61. 
14. Lombardi AV Jr, Berend KR, Adams JB. Why knee replacements fail in 2013: Patient, surgeon or implant? Bone Joint J. 2014; 96-B (11 Supple A): 101-4.

15. Favorito PJ, Mihalko WM, Krackow KA. Total Knee Arthroplasty in the Valgus Knee. J Am Acad Orthop Surg. 2002; 10(1): 1624.

16. Bade M, Kittelson J, Kohrt W, Stevens-Lapsley J. Predicting functional performance and range of motion outcomes after total knee arthroplasty. Am J Phys Med \& Rehabil. 2014; 93(7): 579-85.

17. Lanshammar K, Ribom E. Differences in muscle strength in dominant and non-dominant leg in females aged 20-39 years - a population based study. Phys Ther Sport. 2011; 12(2): 76-9.

18. Young P, Whitall J, Bair W, Rogers M. Leg preference associated with protective stepping responses in older adults. Clin Biomech (Bristol, Avon). 2013; 28(8): 927-32.

19. Shawen S, Belmont P, Klemme W, Topoleski L, Orchowski J. Osteoporosis and anterior femoral notching in periprosthetic supracondylar fractures: a biomechanical analysis. J Bone Joint Surg Am. 2003; 85(1): 115-21.

20. Lesh M, Schneider D, Deol G, Davis B, Jacobs C, Pellegrini V. The consequences of anterior femoral notching in total knee arthroplasty: a biomechanical study. J Bone Joint Surg Am. 2000; 82(8): 1096-101.

21. Ritter M, Thong A, Keating E, Faris P, Meding J, Berend M et al. The effect of femoral notching during total knee arthroplasty on the prevalence of postoperative femoral fractures and on clinical outcome. J Bone Joint Surg Am. 2005; 87(11): 2411-4.

22. Jethanandani R, Patwary M, Shellito A, Meehan J, Amantullah D. Biomechanical consequences of anterior femoral notching in cruciate-retaining versus posterior stabilized total knee arthroplasty. Am J Orthop (Belle Mead NJ). 2016; 45(5): 268-72. 